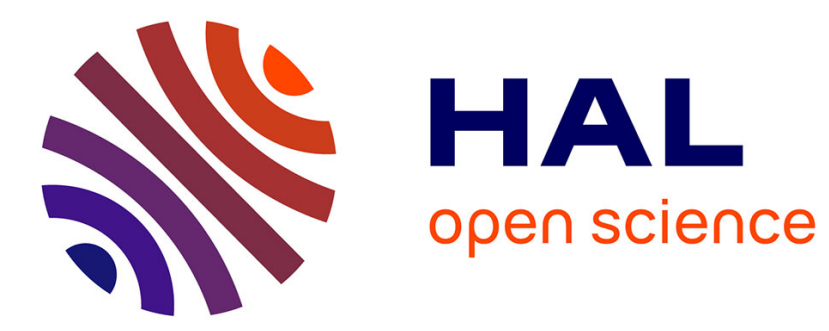

\title{
Hybrid formalism for consensus of a general class of multi-agent systems with biased measurements
}

Tommaso Borzone, Irinel-Constantin Morarescu, Marc Jungers, Michael Boc, Christophe Janneteau

\section{- To cite this version:}

Tommaso Borzone, Irinel-Constantin Morarescu, Marc Jungers, Michael Boc, Christophe Janneteau. Hybrid formalism for consensus of a general class of multi-agent systems with biased measurements. 17th European Control Conference, ECC'19, Jul 2019, Naples, Italy. hal-02169625

\section{HAL Id: hal-02169625 \\ https://hal.science/hal-02169625}

Submitted on 1 Jul 2019

HAL is a multi-disciplinary open access archive for the deposit and dissemination of scientific research documents, whether they are published or not. The documents may come from teaching and research institutions in France or abroad, or from public or private research centers.
L'archive ouverte pluridisciplinaire HAL, est destinée au dépôt et à la diffusion de documents scientifiques de niveau recherche, publiés ou non, émanant des établissements d'enseignement et de recherche français ou étrangers, des laboratoires publics ou privés. 


\title{
Hybrid formalism for consensus of a general class of multi-agent systems with biased measurements
}

\author{
T. Borzone*,, I.-C. Morărescu*, M. Jungers*, M. Boc ${ }^{\dagger}$ and C. Janneteau
}

\begin{abstract}
The paper presents a hybrid formalism to analyze a decentralized control policy for a network of agents characterized by a class of non-linear heterogeneous dynamics and biased measurements. Each agent has a continuous-time dynamics and tracks a piecewise constant impulsive reference. The reference jumps depend on the relative distance with respect to some time-varying neighbors. The jumps take place at some updating instants that are decided independently by each agent. Our results provide input-to-state stability guarantees as far as a minimum and maximum dwell-time condition between consecutive updates is satisfied. A numerical example inspired by the formation realization problem for non-holonomic vehicles illustrates the theoretical results.
\end{abstract}

\section{INTRODUCTION}

In this work we present and analyze a general decentralized control strategy for a class of nonlinear multi-agents systems which cooperate to reach a specific global goal. The proposed algorithm follows the idea in [1] in which agents sense sporadically other agents in their neighborhood and, based on their relative measurements, they update their references. Between two updates of the reference, which is computed in a decentralized manner, the dynamics of each agent becomes completely decoupled from the motion of the other agents in the network (see Fig. 1).

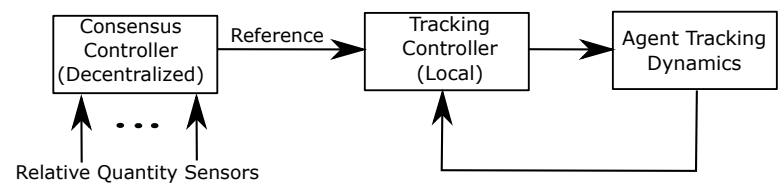

Fig. 1. Block diagram showing the control strategy for each agent $i$. For Local we mean implemented as a separate task and independent from the planning Decentralized level which uses the sensing information about the neighborhood of agent $i^{t h}$.

The decentralized part is modelled by one choice of a discrete agreement algorithm which are largely studied in the literature (see for instance [2], [3]). While many results on this problem already exist, the agents are often assumed to be very simple, being modelled as single or double integrators [4], [5]. In many real applications the agents which compose a network are heterogeneous and have non-linear dynamics

This work was partially funded by the Région Grand Est and CEA Tech Metz.

* T. Borzone, I.-C. Morărescu and M. Jungers are with Université de Lorraine, CNRS, CRAN, F-54000 Nancy, France, (tommaso.borzone, constantin.morarescu, marc.jungers) duniv-lorraine.fr

$\dagger$ T. Borzone, M. Boc and C. Janneteau are with CEA LIST (Commissariat à l'Energie Atomique et aux Energies alternatives - Communicating Systems Laboratory), (tommaso.borzone, michael.boc, christophe.janneteau) dcea.fr which are nontrivial to control, take for example mobile ground robots with non-holonomic dynamics studied by [6], [7] or other class of aerial or submarine vehicles. Consequently, various consensus algorithms for different classes of nonlinear systems received an increasing attention, see for example the review [8] and the references therein.

The decentralized strategy proposed in this work results in a hybrid closed-loop dynamics due to the jumping (nonsmooth) references that agents have to track. Basically, we generalize the results in [9], [10] considering a general class of non-linear systems, taking in account the sensor noise and developing a completely decentralised policy for the choice of the dwell-time between two consecutive communications. We also notice that a similar strategy has been used in [1] to solve the consensus problem for a general class of discretetime nonlinear dynamics.

The main contributions of this paper are related to the consideration of: heterogenous agents dynamics belonging to a generic class of continuous-time nonlinear systems, biased or noisy measurements and asynchronous updates of the references. Two important aspects of the proposed strategy, rendering it implementable on real devices, are the sporadic sensing (unlike [11], [12] where it is assumed in continuous time) of the neighboring agents and the directionality of the interactions between the agents (unlike [13] where the same problem is addressed considering undirected interactions). Another important implementation aspect highlighted in Fig. 1 is that the updates of the references are based on the relative positioning sensors (that may provide biased information) and not on the usage of the neighbors references that would require direct or cloud based communication between agents (as it has been done for example in [14], [15] where, by the way, the agents dynamics are simple or double integrators). Indeed these kinds of communication can be costly and not reliable. Typical examples are the submarine environment where communications via acoustic modem are difficult or in multi UGV (Unmanned Ground Vehicle) scenarios where the exchange of information can be subject to severe interferences depending on the environment. On the contrary, equipping the subsystems with relative sensors (e.g relative positioning range and bearing sensors or sonar and Doppler sensors), would not require wireless communications. Nevertheless, in our algorithm, this comes with a supplementary cost of hybrid modelling of the closedloop dynamics.

In section II we present the class of non-linear systems which describes the agents dynamics and the properties of the network; afterward we introduce the two stage strategy 
used to solve the problem as described in Fig. 1. The nature of the chosen control strategy motivates a new stability and robustness analysis in section III, followed by a brief application example and the conclusions.

\section{A. Notation}

The following standard notation has been used throughout the paper. The symbols $\mathbb{N}, \mathbb{R}$ and $\mathbb{R}_{+}$stand respectively for the set of non-negative integers, the set of real and non-negative real numbers. Given a vector $x$ we denote by $\|x\|,|x|$ its Euclidean and infinity norms, respectively. For a vector $x$ parameterized in time $t \in \mathbb{R}_{+}$we define $|x|_{\left[t_{0}: t\right]}=\sup _{t_{0} \leq \tau \leq t}|x(\tau)|$. The transpose of a matrix $A$ is denoted by $A^{\top}$. The notation $A>0(A \geq 0)$ is used for a matrix with positive (nonnegative) entries; so the symbols $>$ and $<$ are used to represent element-wise inequalities. The $n \times n$ identity matrix is denoted $\mathbf{I}_{n}$ and $x\left(t_{k}^{-}\right)=\lim _{t \rightarrow t_{k}, t \leq t_{k}} x(t)$. The vector of dimension $n$ with all components equal 1 is denoted as $\mathbb{1}_{n}$. A continuous function $\gamma:[0, \alpha) \rightarrow[0, \infty)$ is said to belong to class $\mathcal{K}$ if it is strictly increasing and $\gamma(0)=0$. It is said to belong to class $\mathcal{K}_{\infty}$ if it belongs to class $\mathcal{K}$ and in addition $\alpha=\infty$ and $\gamma(r) \underset{r \rightarrow \infty}{\longrightarrow} \infty$. A continuous function $\beta:[0, \alpha) \times[0, \infty) \rightarrow[0, \infty)$ is said to belong to class $\mathcal{K} \mathcal{L}$ if for each $s, \beta(r, s) \in \mathcal{K}$ w.r.t. $r$ and, for a fixed $r, \beta(r, s) \rightarrow 0$ as $s \rightarrow \infty$.

\section{PRELIMINARIES}

\section{A. Problem Formulation}

We define a set of $N$ agents and we associate to each one a state $x_{i} \in \mathbb{R}^{n}$. We assume that the dynamics of the $i^{t h}$ system in the network is described by the following differential equations

$$
\dot{x}_{i}(t)=\varphi_{i}\left(x_{i}(t), u_{i}(t)\right),
$$

where $u_{i} \in \mathbb{R}^{s}$ is the control action and $\varphi_{i}: \mathbb{R}^{n} \times \mathbb{R}^{s} \rightarrow \mathbb{R}^{n}$ is assumed to be locally Lipschitz. To simplify the presentation we remove the argument $t$ when it is not explicitly needed.

Throughout the paper we denote by $r_{i} \in \mathbb{R}^{n}$ the reference that agent $i$ has to track and by $e_{i} \in \mathbb{R}^{n}$ the associated error coordinates

$$
e_{i}=x_{i}-r_{i}
$$

We impose the following assumptions on the dynamics of each agent.

Assumption 1 (Error Dynamics): For all $r_{i}$ such that $\dot{r}_{i}=$ 0 there exists a locally Lipschitz function $f_{i}: \mathbb{R}^{n} \times \mathbb{R}^{s} \rightarrow \mathbb{R}^{m}$ such that

$$
\dot{e}_{i}=f_{i}\left(e_{i}, u_{i}\right),
$$

Assumption 2 (Exponential Convergence of $e_{i}$ ): There exists $u_{i}=\kappa\left(e_{i}\right)$ such that, considering the closed loop dynamics $\dot{e}_{i}=f_{i}\left(e_{i}, \kappa\left(e_{i}\right)\right)$,

$$
\left|e_{i}(t)\right| \leq c_{i}\left|e_{i}\left(t_{0}\right)\right| \mathrm{e}^{-\lambda_{i}\left(t-t_{0}\right)}, \quad \text { for } t \geq t_{0} .
$$

Remark 1: The first assumption states that as far as the reference is constant there must exist a set of differential equations directly related to the error coordinates, described by the map $f_{i}$. This is the case if we consider dynamics
(1) with $r_{i}=0$ and $f_{i}=\varphi_{i}$. It is also noteworthy that non-holonomic dynamics satisfy Assumptions 1-2 for what concerns the $x-y$ 2D positioning errors as it has been studied in [10] and [9].

We also assume that the agents interact over a directed and time-varying network topology described by the digraph $\mathcal{G}(t)=(\mathcal{V}, \mathcal{E}(t))$, where the vertex-set $\mathcal{V}$ represents the set of agents and the edge set $\mathcal{E}(t) \subset \mathcal{V} \times \mathcal{V}$ collects the interactions between agents at time $t$.

Definition 1: A path of length $l$ in a digraph $\mathcal{G}=(\mathcal{V}, \mathcal{E})$ is a union of directed edges $\bigcup_{k=1}^{l}\left(i_{k}, j_{k}\right)$ such that $i_{k+1}=$ $j_{k}, \forall k \in\{1, \ldots, l-1\}$. The node $j$ is connected with node $i$ in $\mathcal{G}=(\mathcal{V}, \mathcal{E})$ if there exists at least a path in $\mathcal{G}$ from $i$ to $j$ (i.e. $i_{1}=i$ and $j_{l}=j$ ). A connected digraph is such that any of its two distinct elements are connected.

Since we deal with a decentralized policy with sporadic state updates, we introduce the sequence of update instants as

$$
\mathcal{T}=\left\{t_{k}: t_{k} \in \mathbb{R}_{+}, t_{k}<t_{k+1}, \forall k \in \mathbb{N}, \lim _{k \rightarrow \infty} t_{k}=\infty, t_{0}=0\right\} .
$$

We associate to the digraph $\mathcal{G}\left(t_{k}\right)=\left(\mathcal{V}, \mathcal{E}\left(t_{k}\right)\right)$ a row stochastic matrix $P\left(t_{k}\right) \in \mathbb{R}^{N \times N}$ i.e. for all $t_{k} \in \mathcal{T}$

$$
\left\{\begin{array}{l}
P_{i, j}\left(t_{k}\right)=0, \text { if }(i, j) \notin \mathcal{E}\left(t_{k}\right), \\
P_{i, i}\left(t_{k}\right)>b, \forall i=\{1, \ldots, N\}, \\
P_{i, j}\left(t_{k}\right)>a, \text { if }(i, j) \in \mathcal{E}\left(t_{k}\right), \\
\sum_{j=1}^{N} P_{i, j}\left(t_{k}\right)=1, \forall i=\{1, \ldots, N\} .
\end{array}\right.
$$

Throughout the paper we impose the following assumptions.

Assumption 3 (Bounded Intercommunication Interval):

If $i$ communicates with $j$ an infinite number of times (that is, if $(i, j) \in \mathcal{E}\left(t_{k}\right)$ infinitely often), then there is some $L \in \mathbb{N}$ such that, for all $t_{k} \in \mathcal{T}$, $(i, j) \in \mathcal{E}\left(t_{k}\right) \cup \mathcal{E}\left(t_{k+1}\right) \cup \cdots \cup \mathcal{E}\left(t_{k+L-1}\right)$.

Assumption 4 (Minimal Influence): There exists $a \in$ $(0,1)$ and $b \in\left(\frac{n L-1}{n L}, 1\right)$ such that, $\forall t_{k} \in \mathcal{T}, P_{i, i}\left(t_{k}\right) \geq b$ and, if $P_{i, j}\left(t_{k}\right) \neq 0$ and thus $(i, j) \in \mathcal{E}\left(t_{k}\right)$ then $P_{i, j}\left(t_{k}\right)>a$.

Assumption 5 (Joint Connectivity): $\overline{\mathcal{G}}=\cup_{k \geq k_{0}} \mathcal{G}\left(t_{k}\right)$ is strongly connected for all $k_{0} \in \mathbb{N}$.

Remark 2: These three Assumptions are usually formulated to prove the convergence of the consensus algorithm for general networks (see for example [16], [17]). In particular in Assumption 4 we provide a lower bound for $b$ whose value directly follows from the proof of Theorem 2 in the following.

Finally, we define $\mathcal{T}_{i}$ as the infinite countable subset of $\mathcal{T}$ collecting the update instants only for the agent $i$. Formally

$$
\mathcal{T}_{i}=\left\{t_{k} \in \mathcal{T} \mid \exists j \in \mathcal{V},(i, j) \in \mathcal{E}\left(t_{k}\right)\right\}
$$

Using the definition of $\mathcal{T}_{i}$ we introduce the last update which affected the agent $i$ as $\underline{t}_{k, i}=\sup \left\{t_{h} \in \mathcal{T}_{i} \mid h<k\right\}$. We then assume the following for all the agents.

Assumption 6 (Dwell Time): There exist positive constants $\underline{\tau}, \bar{\tau}>0$ such that, for all $t_{k} \in \mathcal{T}_{i}$

$$
\underline{\tau} \leq t_{k}-\underline{t}_{k, i} \leq \bar{\tau}, \quad \forall i \in\{1, \ldots, N\} .
$$


In other words, there exist a lower and an upper bound for the time elapsed between two consecutive updates of the same agent.

To describe the overall behavior of the network we introduce the collective vectors $r=\left(r_{1}^{\top}, \cdots, r_{N}^{\top}\right)^{\top}, e=$ $\left(e_{1}^{\top}, \cdots, e_{N}^{\top}\right)^{\top}$ and $x=\left(x_{1}^{\top}, \cdots, x_{N}^{\top}\right)^{\top}$ belonging $\mathbb{R}^{n \cdot N}$ and related by (2).

The updates are defined by a map $g_{i}: \mathbb{R}^{N \times n} \rightarrow \mathbb{R}^{n}$ which describes a general decentralized algorithm:

$$
r_{i}\left(t_{k}\right)=g_{i}\left(x\left(t_{k}^{-}\right)\right)+\delta_{i}\left(t_{k}^{-}\right), \text {for } t_{k} \in \mathcal{T}_{i},
$$

where we assume that we are able to model the impact of the measurements error as an additive noise $\delta_{i}$, bounded by $\bar{\delta}=\left|\delta_{i}\right|_{[0: \infty]}, \forall i \in\{1, \ldots, N\}$.

It is worth notting that the error $e_{i}$ is also affected by the jumps. Indeed, following (2) the jumps propagate to the errors through $e_{i}\left(t_{k}\right)=x_{i}\left(t_{k}^{-}\right)-r_{i}\left(t_{k}\right)$.

Collecting together the dynamics of the systems during the continuous time and during the reference update instants leads to the following hybrid formalization for all $i \in$ $\{1, \ldots, N\}$ (see [18] for an exhaustive presentation of hybrid dynamics)

$$
\left\{\begin{array}{l}
\dot{r}_{i}(t)=0 \\
\dot{e}_{i}(t)=f_{i}\left(e_{i}(t), \kappa\left(e_{i}(t)\right)\right.
\end{array}\right.
$$

for all $t \in \mathbb{R}^{+}-\mathcal{T}_{i}$ and

$$
\left\{\begin{array}{l}
r_{i}\left(t_{k}\right)=g_{i}\left(r\left(t_{k}^{-}\right)+e\left(t_{k}^{-}\right)\right)+\delta_{i}\left(t_{k}^{-}\right) \\
e_{i}\left(t_{k}\right)=e_{i}\left(t_{k}^{-}\right)+r_{i}\left(t_{k}^{-}\right)-g_{i}\left(e\left(t_{k}^{-}\right)+r\left(t_{k}^{-}\right)\right)+\delta_{i}\left(t_{k}^{-}\right)
\end{array}\right.
$$

for all $t_{k} \in \mathcal{T}_{i}$, where for consistency we impose $\delta_{i}\left(0^{-}\right)=$ $0, e\left(0^{-}\right)=0, r\left(0^{-}\right)=x(0)$.

Remark 3: Note that the flow dynamics (7) is completely decentralized meaning that each agent tracks its reference and no interaction with other agents is required in continuous time.

\section{B. Decentralized Control Strategy and Analysis}

The objective of this paper is to design and analyze a decentralized algorithm allowing the agents to reach an agreement on the value of their state. Since we assume the agents can track exponentially fast a constant reference we will focus on the design of a jump map $g_{i}$ as defined in (6). This can be done in a decentralized way by applying a discrete-time linear consensus algorithm $\forall t_{k} \in \mathcal{T}$ :

$$
r_{i}\left(t_{k}\right)=x_{i}\left(t_{k}^{-}\right)+\sum_{j \neq i} P_{i, j}\left(t_{k}\right)\left(x_{j}\left(t_{k}^{-}\right)-x_{i}\left(t_{k}^{-}\right)\right)+\delta_{i}\left(t_{k}^{-}\right) \text {. }
$$

We now introduce some quantities useful for the following analysis.

Definition 2: With $m=\left(\min _{i \in \mathcal{V}} r_{i_{1}}, \cdots, \min _{i \in \mathcal{V}} r_{i_{n}}\right)^{\top}$ and $M=\left(\max _{i \in \mathcal{V}} r_{i_{1}}, \cdots, \max _{i \in \mathcal{V}} r_{i_{n}}\right)^{\top}$ we define the vectors which collect minimum and the maximum among the same components of vectors $r_{i}$. The difference between these maximum and minimum vectors will be called diameter and denoted by $\Delta=M-m$. Finally with $m_{h}, M_{h}$, and $\Delta_{h}$ we will denote the $h^{\text {th }}$ component of the corespondent vector.
Remark 4: Note that the jump map (9) of one agent requires only information from the neighbors (in the interconnection graph). Indeed, following conditions (5), if no sensing information is available for agent $j$, the corresponding element $P_{i, j}\left(t_{k}\right)=0$.

The aim of the paper is to analyze the stability properties of the set

$$
\mathcal{A}=\left\{e, r \in \mathbb{R}^{n \cdot N}: e=0, \Delta=0\right\},
$$

for the $N$ interconnected hybrid systems (7)-(8) with the chosen decentralized control (9). The set in (10) requires that each agent reaches its own reference $(e=0)$ and that all the references achieve consensus over all the $n$ components $(\Delta=0)$.

In the following, in order to prove the convergence of the proposed strategy we reduce the analysis of the interconnected hybrid dynamics in (7)-(8) to the analysis of a hybrid positive system with constant input $d(t)=\bar{\delta}, \forall t \in \mathbb{R}_{+}$with the following dynamics

$$
\begin{array}{lc}
\dot{q}(t)=f_{q}(q(t)), & \text { for } t \in \mathbb{R}^{+}-\mathcal{T}, \\
q\left(t_{k}\right)=g_{q}\left(q\left(t_{k}^{-}\right)\right)+d\left(t_{k}^{-}\right), & \text {for } t_{k} \in \mathcal{T},
\end{array}
$$

where $q=\left(\Delta^{\top},\left|e_{1}\right|, \ldots,\left|e_{N}\right|\right)^{\top} \in \mathbb{R}_{+}^{p}$, and $f_{q}: \mathbb{R}_{+}^{p} \rightarrow \mathbb{R}_{+}^{p}$ and $g_{q}: \mathbb{R}_{+}^{p} \times \mathbb{R}_{+} \rightarrow \mathbb{R}_{+}^{p}$ are continuous with $p=n+N$.

With this choice of state variable, the asymptotic properties of $\mathcal{A}$ are equivalent to the ones of the origin $q=0$ with respect to dynamics (11)-(12). We define two basic asymptotic properties that are of interest for our system (see [19], [20]). The first concerns the discrete-time trajectory of the systems, and consequently the solution $q\left(t_{k}\right)$ is parametrized in $t_{k} \in \mathcal{T}$. The second property characterizes the overall hybrid trajectory of the system and so the solution $q\left(t, t_{k}\right)$ is parametrized in both $t \in \mathbb{R}_{+}$and $t_{k} \in \mathcal{T}$.

Definition 3: The discrete dynamics (12) is input-to-state stable, ISS with respect to the input $d$ and the origin $q=0$ if there exists a function $\beta$ of class $\mathcal{K} \mathcal{L}$ and a function $\gamma$ of class $\mathcal{K}_{\infty}$ such that

$$
\left|q\left(t_{k}\right)\right| \leq \beta(|q(0)|, k)+\gamma\left(|d|_{\left[0: t_{k}\right]}\right), \quad \forall t_{k} \in \mathcal{T} .
$$

Definition 4: The hybrid system (11)-(12) is input-to-state stable, ISS with respect to the input $d$ and the origin $q=0$ if there exist a function $\tilde{\beta}$ of class $\mathcal{K} \mathcal{L}$ and a function $\tilde{\gamma}$ of class $\mathcal{K}_{\infty}$ such that

$$
\left|q\left(t, t_{k}\right)\right| \leq \tilde{\beta}(|q(0,0)|, t+k)+\tilde{\gamma}\left(|d|_{\left[0: t_{k}\right]}\right),
$$

$\forall t_{k} \in \mathcal{T}, t \in\left[t_{k}, t_{k+1}\right)$

\section{Stability And Robustness}

This section starts with the analysis of the discrete evolution of $q$. Precisely, we show that (12) is ISS with respect to $q=0$. Then, we extend the result to the whole hybrid system (11)-(12) with special focus on the ISS property in order to characterize the robustness of the control strategy. 


\section{A. Bounds on the jump dynamics}

Thanks to Assumption 2 the agents converge toward their reference during the flow (i.e. $e_{i} \rightarrow 0$ ) but the reference $r_{i}$ remains constant.

We start by investigating the behavior of the discrete part of the hybrid system (8). Moreover, by rewriting the jump dynamics (9) in terms of $r_{i}$ and $e_{i}$ one obtains for $t_{k} \in \mathcal{T}$

$$
\begin{aligned}
& r_{i}\left(t_{k}\right)=\sum_{j=1}^{N} P_{i, j}\left(t_{k}\right)\left(e_{j}\left(t_{k}^{-}\right)+r_{j}\left(t_{k}^{-}\right)\right)+\delta_{i}\left(t_{k}^{-}\right) \\
& e_{i}\left(t_{k}\right)=e_{i}\left(t_{k}^{-}\right)+r_{i}\left(t_{k}^{-}\right)-\sum_{j=1}^{N} P_{i, j}\left(t_{k}\right)\left(e_{j}\left(t_{k}^{-}\right)+r_{j}\left(t_{k}^{-}\right)\right)+\delta_{i}\left(t_{k}^{-}\right) .
\end{aligned}
$$

The aim of this section is to prove that after at most $L$ updates the values of the references diameters $\Delta$ and the errors norms $\left|e_{i}\right|$ decrease as far as the measurement bias is small enough.

We start with two instrumental results concerning the update of the reference (15). Recalling Definition 2, the result of the following Lemma shows that the update of the minimum $m$ and of the maximum $M$ of the references are respectively lower and upper bounded.

Lemma 1: Under Assumptions 3-5, for all $t_{k} \in \mathcal{T}$, the following holds

$$
m\left(t_{k}\right) \geq m\left(t_{k-1}\right)-\sum_{j=1}^{N}\left|e_{j}\left(t_{k}^{-}\right)\right| \mathbb{1}_{n}-\bar{\delta} \mathbb{1}_{n}
$$

and

$$
M\left(t_{k}\right) \leq M\left(t_{k-1}\right)+\sum_{j=1}^{N}\left|e_{j}\left(t_{k}^{-}\right)\right| \mathbb{1}_{n}+\bar{\delta} \mathbb{1}_{n} .
$$

The next Lemma is essentially a generalisation of the previous one, which concerns the bound over the reference update of any agent $i$ in the network.

Lemma 2: Let Assumptions 3-5 hold. Then for all $(\bar{l}, \bar{J}) \in$ $\mathcal{E}\left(t_{k}\right), t_{k} \in \mathcal{T}_{i}$, the following holds

$$
r_{\bar{l}}\left(t_{k}\right) \geq m\left(t_{k}^{-}\right)+a\left(r_{\bar{J}}\left(t_{k}^{-}\right)-m\left(t_{k}^{-}\right)\right)-\sum_{j=1}^{N}\left|e_{j}\left(t_{k}^{-}\right)\right| \mathbb{1}_{n}-\bar{\delta} \mathbb{1}_{n}
$$

and

$$
r_{\bar{l}}\left(t_{k}\right) \leq M\left(t_{k}^{-}\right)-a\left(M\left(t_{k}^{-}\right)-r_{\bar{J}}\left(t_{k}^{-}\right)\right)+\sum_{j=1}^{N}\left|e_{j}\left(t_{k}^{-}\right)\right| \mathbb{1}_{n}+\bar{\delta} \mathbb{1}_{n} .
$$

Now we recursively extend the result in Lemma 2 to the last $L$ updates that occurred in the network (recalling that $L$ represents the maximum number of updates that occur in the network between two activations of any $(i, j) \in \mathcal{E}$, see Assumption 3). The following result is inspired by Lemma 3.2 in [17].

Lemma 3: Let Assumptions 3-5 hold. Then, given $\bar{\imath} \in \mathcal{V}$, for all $i \in \mathcal{V}$, and for all $k>L, k \in \mathbb{N}$ we have

$$
\begin{aligned}
r_{i}\left(t_{k}\right) \geq & m\left(t_{k-L}\right)+a^{L}\left(r_{\bar{l}}\left(t_{k-L}\right)-m\left(t_{k-L}\right)\right)- \\
& \sum_{j=1}^{N} \sum_{l=0}^{L-1}(2-a) a^{l}\left|e_{j}\left(t_{k-l}^{-}\right)\right| \mathbb{1}_{n}-\sum_{l=0}^{L-1}(2-a) a^{l} \bar{\delta} \mathbb{1}_{n}
\end{aligned}
$$

and

$$
\begin{aligned}
r_{i}\left(t_{k}\right) \leq & M\left(t_{k-L}\right)-a^{L}\left(M\left(t_{k-L}\right)-r_{\bar{l}}\left(t_{k-L}\right)\right)+ \\
& \sum_{j=1}^{N} \sum_{l=0}^{L-1}(2-a) a^{l}\left|e_{j}\left(t_{k-l}^{-}\right)\right| \mathbb{1}_{n}+\sum_{l=0}^{L-1}(2-a) a^{l} \bar{\delta} \mathbb{1}_{n} .
\end{aligned}
$$

Exploiting the exponential decreasing of the error in continuous time according to Assumption 2 and defining $c=\max _{j \in \mathcal{V}} c_{j}$ and $\lambda=\min _{j \in \mathcal{V}} \lambda_{j}$ we get the following result.

Proposition 1: Under Assumptions 1-6, there exist $\eta_{1}, \eta_{2} \in \mathbb{R}_{+}$such that for all $k>2 L, k \in \mathbb{N}$ one has

$\Delta\left(t_{k}\right) \leq \eta_{1} \Delta\left(t_{k-L}\right)+\sum_{j=1}^{N} \sum_{l=0}^{2 L-1} \eta_{2} c \mathrm{e}^{-\lambda \underline{\tau}}\left|e_{j}\left(t_{k-l-1}\right)\right| \mathbb{1}_{n}+\sum_{l=0}^{L-1} \eta_{2} a^{l} \bar{\delta} \mathbb{1}_{n}$.

Moreover, possible values for $\eta_{1}, \eta_{2}$ are $\eta_{1}=\left(1-a^{L}\right), \eta_{2}=$ $2(2-a)$.

As it has been done for the reference diameters we analyze the update law of the error (16). The following Proposition characterizes the error norm evolution.

Proposition 2: Let Assumptions 1-6 hold. Then there exists $\eta_{3} \in \mathbb{R}_{+}$, such that for all $k>L, k \in \mathbb{N}$ one has

$$
\begin{aligned}
\left|e_{i}\left(t_{k}\right)\right| \leq & \sum_{l=0}^{L-1} c \mathrm{e}^{-\lambda \underline{\tau}}\left|e_{i}\left(t_{k-l-1}\right)\right|+\sum_{j=1, j \neq i}^{N} \sum_{l=0}^{L-1} \eta_{3} c \mathrm{e}^{-\lambda \underline{\tau}}\left|e_{j}\left(t_{k-l-1}\right)\right| \\
& +\sum_{l=0}^{L-1} \eta_{3} \mathbb{1}_{n}^{\top} \Delta\left(t_{k-l-1}\right)+\bar{\delta}
\end{aligned}
$$

for all $i=\{1, \ldots, N\}$. Moreover a possible value is $\eta_{3}=$ $(1-b)$.

Let us remark that until now, thanks to Proposition 1-2, we obtained $n$ inequalities for the reference diameters and $N$ inequalities for the error norms. To characterize the stability properties of the jumps dynamics we rewrite inequalities (23)-(24) in a more compact form. In the sequel we will make use of the following matrices, whose values depend on $c$ and $\eta_{1}, \eta_{2}, \eta_{3}$ introduced in Propositions 1-2. In particular we define $B_{1}, B_{2}, B_{3}, \Gamma_{1}, \Gamma_{2} \in \mathbb{R}_{+}^{(n+N}$

$B_{1}=\left[\begin{array}{cc}\eta_{1} \mathbf{I}_{n} & 0 \\ 0 & 0\end{array}\right], B_{2}=\left[\begin{array}{cc}0 & 0 \\ 0 & c \mathbf{I}_{N}\end{array}\right] B_{3}=\left[\begin{array}{cc}0 & 0 \\ \eta_{3} \mathbb{1}_{N \times n} & 0\end{array}\right]$,
$\Gamma_{1}=\left[\begin{array}{cc}0 & \eta_{2} c \mathbb{1}_{n \times N} \\ 0 & \eta_{3} c \mathbf{J}_{N}\end{array}\right], \Gamma_{2}=\left[\begin{array}{cc}0 & \eta_{2} c \mathbb{1}_{n \times N} \\ 0 & 0\end{array}\right]$

where the matrix $\mathbf{J}_{N}=\left(\mathbb{1}_{N \times N}-\mathbf{I}_{N}\right)$. We use these blocks to condense the results expressed in Propositions 1-2 in the following lemma.

Lemma 4: Under Assumptions 1-5, $\forall t_{k} \in \mathcal{T}, k>2 L$, one has that:

$$
\bar{q}\left(t_{k}\right) \leq\left(B+\Gamma \mathrm{e}^{-\lambda \underline{\tau}}\right) \bar{q}\left(t_{k-1}\right)+G \bar{d}\left(t_{k-1}\right),
$$


where $B, \Gamma \in \mathbb{R}_{+}^{p \times p}, G \in \mathbb{R}_{+}^{p \times L}$ and $\bar{q} \in \mathbb{R}_{+}^{p}, p=(N+n) \cdot 2 L$, with structures

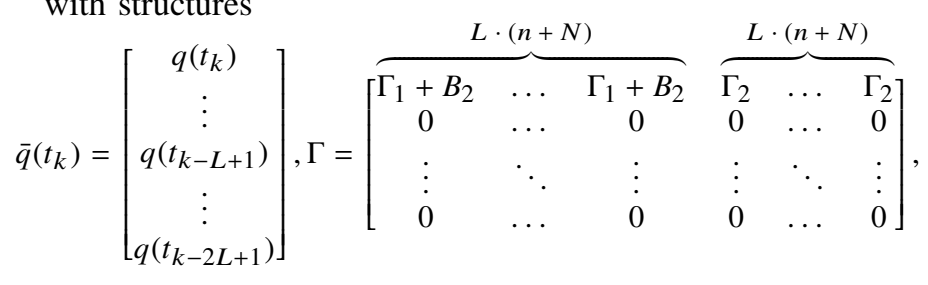

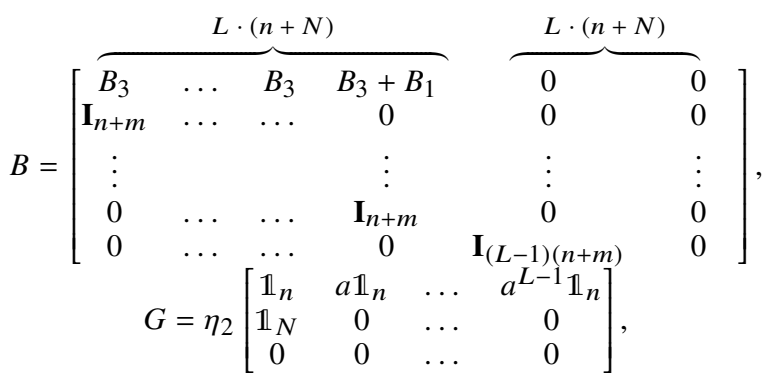

and $\bar{d} \in \mathbb{R}^{L}, d\left(t_{k}\right)=\bar{\delta} \mathbb{1}_{L}$ for all $t_{k} \in \mathcal{T}$.

\section{B. Input-to-state stability of the jumps dynamics}

The following result formalizes the implication of dynamics (26) on the stability of the origin $q=0$ with the chosen decentralized control (9).

Theorem 1: Under Assumptions 1-6, let $\underline{\tau}^{*}>0$ such that the positive matrix $B+\Gamma \mathrm{e}^{-\lambda \underline{\tau}^{*}}$ is Schur. Then, for all sequences $\mathcal{T}=\left(t_{k}\right)_{k \geq 0}$ of jump times satisfying the condition $\underline{\tau} \geq \underline{\tau}^{*}, q=0$ is globally asymptotically stable with respect to the jumps dynamics (8) for $d \equiv 0$. Furthermore, the jumps dynamics (8) is ISS according to Definition 3, or equivalently there exists $\theta, \gamma_{d}>0$ such that

$$
\left|q\left(t_{k}\right)\right| \leq \mathrm{e}^{-\theta k}|q(0)|+\gamma_{d}|d|_{\left[0: t_{k}\right]}, \quad \forall t_{k} \in \mathcal{T} .
$$

Proof: Since $\underline{\tau} \geq \underline{\tau}^{*}$, if such a $\underline{\tau}^{*}$ exists and the matrix $B+\Gamma \mathrm{e}^{-\lambda \underline{\tau}^{*}}$ is Schur, then $\bar{q}=0$ is globally asymptotically stable for the dynamics (26) and so it is $q=0$. This fact, together with the fact that the input is bounded by $|d|_{\left[0: t_{k}\right]} \leq$ $\bar{\delta}$ imply the existence of a $\theta, \gamma_{d}$ such that condition (28) is true (take for example $\mathrm{e}^{-\theta}=\rho\left(B+\Gamma \mathrm{e}^{-\lambda \underline{\tau}^{*}}\right.$ ) where $\rho(A)$ is the spectral radius of matrix $A$ ).

In the following we provide a value $\tau^{*}$ rendering the matrix $B+\Gamma \mathrm{e}^{-\lambda \underline{\tau}^{*}}$ Schur as in the statement of Theorem 1.

Theorem 2: Let Assumptions 1-5 hold. A sufficient condition that ensures (8) is ISS with respect to $d$ and $q=0$ is that $\underline{\tau} \geq \underline{\tau}^{*}$ with

$\underline{\tau}^{*}=\frac{1}{\lambda} \ln \max \left\{\frac{\eta_{2} c N}{1-\eta_{1} \alpha^{-(L-1)}} \sum_{l=0}^{2 L-1} \alpha^{-l}, \frac{c\left(\eta_{3}(N-1)+1\right)}{1-\eta_{3} n L \alpha^{-(L-1)}} \sum_{l=0}^{L-1} \alpha^{-l}\right\}$,

and $\forall i \in\{1, \ldots, N\}$, with $\alpha$ such that $\max \left\{\eta_{1}^{\frac{1}{L-1}}, \eta_{3} n L^{\frac{1}{L-1}}\right\}<\alpha<1$ and $0<\alpha<1$ for $L=1$.

Remark 5: Note that Theorem 2 does not define the instants of references updates but it gives a lower bound between two consecutive updates for all the agents $i$ independently. Note also that the existence of $\alpha$ is guaranteed thanks to Assumption 4.

\section{Overall hybrid system input-to-state stability}

Now we complete the analysis of the asymptotic properties with our second main result which concerns the input-to-state stability of the overall positive hybrid system (11)-(12). To do so we first introduce the following Lemma that proves the exponential decreasing of $q$ during the flow.

Lemma 5: Let Assumptions 1-6 hold. There exist a $K>0$ and an arbitrarily small $\beta_{c}>0$ such that $\beta_{c} \leq \lambda$ and for all $t_{k} \in \mathcal{T}$

$$
|q(t)| \leq K \mathrm{e}^{-\beta_{c}\left(t-t_{k}\right)}\left|q\left(t_{k}\right)\right|, \quad \forall t \in\left[t_{k}, t_{k+1}\right) .
$$

Moreover, inequality (30) holds for

$$
K \geq \mathrm{e}^{\beta_{c} \bar{\tau}}+c .
$$

We can finally complete the stability analysis with the following ISS condition for the system (11)-(12) where the trajectories of the state are parametrized in both discrete and continuous time.

Theorem 3: If the sufficient condition in Theorem 2 is met and for all sequences $\mathcal{T}=\left(t_{k}\right)_{k \geq 0}$ of jump times $\bar{\tau} \leq \bar{\tau}^{*}<$ $\frac{\theta}{\beta_{c}}$, then the system (11)-(12) is ISS according to Definition 4. Furthermore, there exist $\beta_{d}, \gamma>0$ such that for all $t_{k} \in \mathcal{T}$ and $t \in\left[t_{k}, t_{k+1}\right)$

$$
\left|q\left(t, t_{k}\right)\right| \leq K \mathrm{e}^{-\beta_{c} t-\beta_{d} k}|q(0,0)|+\gamma|d|_{\left[0: t_{k}\right]} .
$$

\section{APPLICATION AND NumERICAL EXAMPLE}

In this section we consider, as a case of study, a set of agents consisting of $N=5$ non-holonomic robots. The robots have to realize a pentagon formation defined by a set of position $p_{i}$. As it is deeply studied in [10] and [9] the references position $r_{i}=\left(r_{x_{i}}, r_{y_{i}}\right)^{\top}$ of each robot is updated from time to time with a discrete consensus algorithm, resulting in a piece-wise constant reference. The dynamics of the agents is expressed in terms of the the Cartesian $x-y$ positioning error $e_{i}=\left(e_{x_{i}}, e_{y_{i}}\right)^{\top}$ and the orientation error $e_{\theta_{i}}$ as $\dot{e}_{x_{i}}=v_{i} \cos e_{\theta_{i}}, \dot{e}_{y_{i}}=v_{i} \sin e_{\theta_{i}}, \dot{e}_{\theta_{i}}=\omega_{i}$. A discontinuous control law (see [21] for details) for the inputs $v_{i}, \omega_{i}$ guarantees the convergence to zero of the position $e_{i}$ to fulfill Assumption 2. In order to realize the formation, the robots need to reach an agreement over the value of the formation displacement $\tilde{r}_{i}(t)=r_{i}(t)-p_{i}$. We can then already identify the number of components $n=2$.

The interaction between the agents switches quasirandomly (we enforce the intercommunication interval to $L=4$ ) between the ones described by the three graphs represented in Fig. 2 following a switch function $\sigma: \mathcal{T} \rightarrow$ $\{1,2,3\}$. The three digraphs topologies have been chosen such that the union digraph $\mathcal{G}=\mathcal{G}_{1} \cup \mathcal{G}_{2} \cup \mathcal{G}_{3}$ is strongly connected, in order to comply with Assumption 5.

To each digraph corresponds a different weight stochastic matrix $P_{\sigma\left(t_{k}\right)}=P\left(t_{k}\right)$, where the elements $P_{i j}\left(t_{k}\right)$ are chosen locally in order to fulfills the requirements of Assumption 4 taking for instance $b \in\left(\frac{L n-1}{L n}, 1\right)$ and $a=\frac{1-b}{n_{i}}$ where $n_{i}$ is the number of agent linked to $i$ at time $t_{k}$.

A dwell-time $\underline{\tau}^{*}=14.6 \mathrm{~s}$ has been used as lower bound for the intervals between the reset and the following, where the value of $\tau^{*}$ has been evaluated using expression (29). 


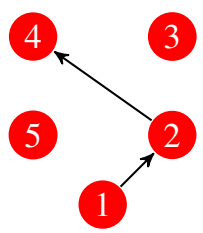

(a) $\sigma\left(t_{k}\right)=1$

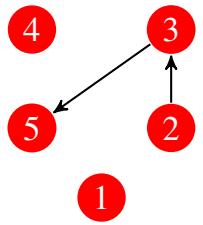

(b) $\sigma\left(t_{k}\right)=2$

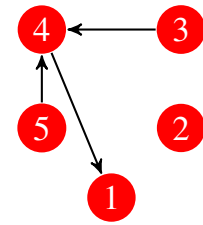

(c) $\sigma\left(t_{k}\right)=3$
Fig. 2. Directed graphs used to implement the sensing interaction.

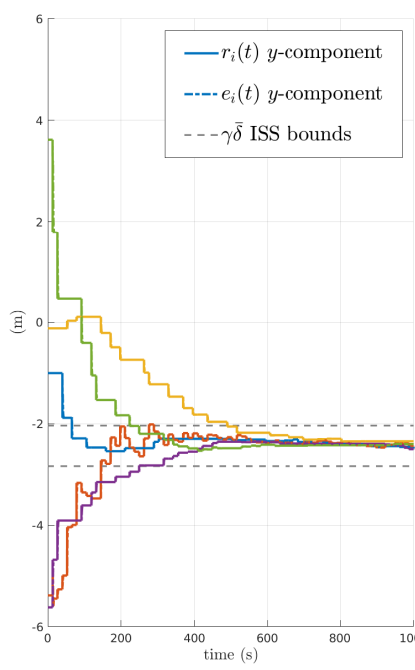

(a)

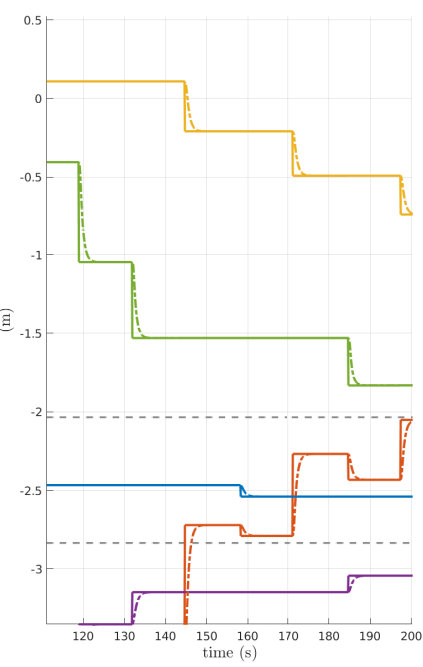

(b)
Fig. 3. In Fig. 3(a) the trajectories of the $y$-component of the $r_{i}$ for each robot together with the positioning error $e_{i}$ are represented. The zoom-in in Fig. 3(b) points out the exponential convergence of the errors between two updates.

The noise coming from the relative positioning sensors is bounded as $\bar{\delta}=0.2 \mathrm{~m}$.

The convergence of the 5 robots towards an agreement over the displacement $\tilde{r}_{i}(t)=r_{i}(t)-p_{i}$, is represented in Fig. 3 where the trajectories of the positioning error $e_{i_{y}}$ is also represented.

\section{Conclusions}

This work proposed the synthesis and the analysis of a decentralized control strategy for networks of non-linear and heterogeneous systems affected by sensor disturbances. The strategy under consideration requires sporadic interactions between agents. These interactions are considered directed and asynchronous. When an interaction occurs an agent updates the reference to track according to its neighbors based on some relative inter-distance measurements affected by a bounded noise. The resulting closed-loop dynamics is hybrid and sufficient ISS conditions are formulated in term of a minimum and maximum dwell-time condition. Finally the case of a fleet of non-holonomic robots realizing a formation is presented as a case of study and numerical example.

\section{REFERENCES}

[1] L. Buşoniu and I.-C. Morărescu, "Consensus for black-box nonlinear agents using optimistic optimization," Automatica, vol. 50, no. 4, pp. 1201-1208, 2014.

[2] J. N. Tsitsiklis, "Problems in decentralized decision making and computation." Massachusetts Inst of Tech Cambridge Lab for Information and Decision Systems, Tech. Rep., 1984.

[3] A. Jadbabaie, J. Lin, and A. S. Morse, "Coordination of groups of mobile autonomous agents using nearest neighbor rules," IEEE Trans. on Automatic Control, vol. 48, no. 6, pp. 988-1001, 2003.

[4] R. Beard and V. Stepanyan, "Information consensus in distributed multiple vehicle coordinated control," in Proceedings of the 42th IEEE Conference on Decision and Control, vol. 2, 2003, pp. 2029-2034.

[5] M. Fiacchini and I.-C. Morărescu, "Convex conditions on decentralized control for graph topology preservation," IEEE Transactions on Automatic Control, vol. 59, no. 6, pp. 1640-1645, DOI.10.1109/TAC.2013.2292719 2014.

[6] Z.-P. Jiang and H. Nijmeijier, "Tracking control of mobile robots: A case study in backstepping." Automatica, vol. 33, no. 7, pp. 13931399, 1997.

[7] C. Samson and K. Ait-Abderrahim, "Feedback control of a nonholonomic wheeled cart in cartesian space." in in Proc. IEEE International Conference on Robotics and Automation, 1991, pp. 1136-1141.

[8] Y. Cao, W. Yu, W. Ren, and G. Chen, "An overview of recent progress in the study of distributed multi-agent coordination," IEEE Transactions on Industrial informatics, vol. 9, no. 1, pp. 427-438, 2013.

[9] T. Borzone, I.-C. Morărescu, M. Jungers, M. Boc, and C. Janneteau, "Hybrid framework for consensus in directed and asynchronous network of non-holonomic agents," IEEE control systems letters, vol. 2, no. 4, pp. 707-712, 2018.

[10] T. Borzone, I.-C. Morărescu, M. Jungers, M. Boc, and C. Janneteau, "Hybrid framework for consensus in fleets of non-holonomic robots," in Proceedings of American Control Conference, 2018.

[11] N. Léchevin, C. A. Rabbath, and P. Sicard, "Trajectory tracking of leader-follower formations characterized by constant line-of-sight angles," Automatica, vol. 42, no. 12, pp. 2131-2141, 2006.

[12] T. Liu and Z.-P. Jiang, "Distributed nonlinear control of mobile autonomous multi-agents," Automatica, vol. 50, no. 4, pp. 1075-1086, 2014.

[13] D. Liuzza, D. V. Dimarogonas, M. Di Bernardo, and K. H. Johansson, "Distributed model based event-triggered control for synchronization of multi-agent systems," Automatica, vol. 73, pp. 1-7, 2016.

[14] A. Adaldo, D. Liuzza, D. V. Dimarogonas, and K. H. Johansson, "Multi-agent trajectory tracking with self-triggered cloud access," in 2016 IEEE 55th Conference on Decision and Control (CDC). IEEE, 2016, pp. 2207-2214.

[15] — - "Cloud-supported formation control of second-order multi-agent systems," IEEE Transactions on Control of Network Systems, 2017.

[16] V. D. Blondel, J. M. Hendrickx, A. Olshevsky, and J. Tsitsiklis, "Convergence in multiagent coordination, consensus, and flocking," in Proc. IEEE Conf. on Decision and Control, 2005, pp. 2996-3000.

[17] D. P. Bertsekas and J. N. Tsitsiklis, Parallel and distributed computation: numerical methods. Prentice hall Englewood Cliffs, NJ, 1989, vol. 23.

[18] R. Goebel, R. Sanfelice, and A. Teel, Hybrid Dynamical Systems. Princeton University Press, 2012.

[19] Z.-P. Jiang and Y. Wang, "Input-to-state stability for discrete-time nonlinear systems," Automatica, vol. 37, no. 6, pp. 857-869, 2001.

[20] C. Cai and A. R. Teel, "Characterizations of input-to-state stability for hybrid systems," Systems \& Control Letters, vol. 58, no. 1, pp. 47-53, 2009.

[21] C. C. de Wit and O. J. Sordalen, "Exponential stabilization of mobile robots with nonholonomic constraints," IEEE Transactions on Automatic Control, vol. 37, no. 11, pp. 1791-1797, 1992. 